\title{
Band Spectra of Diatomic Molecules*
}

$\mathrm{D}$ URING the last ten years, few branches of physical inquiry have called forth a greater output of published work than that of molecular spectra. Many reasons may doubtless be adduced to account for this. On the theoretical side, it is generally recognised that the anharmonic vibratingrotating molecule with its complex magnetic couplings, and the interaction of these energy types with each other and with the electronic energy, provides one of the most fascinating applications of the quantum theory. On the experimental side, æsthetic considerations (and the interest of unravelling and ordering what is often a very complex system of spectral lines) must be given no little weight. Whatever may be the impressions given by a reading of the theory, the experimentalist is well aware that scarcely any band system is really typical or without peculiarities of particular significance for the structure of the emitting molecule.

Further reasons are found also in the variety of other fields of physical inquiry with which this subject has become closely related. The determination of the heats of dissociation of molecules from their spectral data is a well known possibility. The method is applicable to molecular ions and other diatomic molecules incapable of direct thermo-chemical investigation. In another field-that of isotopy-the discovery and quantitative estimation of comparatively rare isotopes of several common elements are well known. The prolific output of work on the spectrum of light scattered by molecules-the Raman effect-has among other things provided a new weapon for studying the solid and liquid states and the vibrations of complex molecular groupings. Molecular spectra have also made a definite contri-

* The Physical Society. "Report on Band-Spectra of Diatomic Molecules". By Dr. W. Jevons. Pp. vi +308 . (London: Physical Socicty, 1932.) 17s. 6d. net. bution to astrophysical knowledge, while photochemical investigation stands clearly in a very close relationship to the subject. The nature of valency and of related topics, which has been clarified in recent years by the work of Heitler, London, and others, provides another link with chemistry.

For a subject with such wide associations, the collected literature has been remarkably scanty, even when we make due allowance for the difficulty of providing it during a period of rapid development. The present writer, faced with the problem of advising men wishing to undertake post-graduate research in this subject as to their reading, has found the answer one of perennial difficulty. The advice given has usually been: "Read certain sections of the National Research Council Bulletin on 'Molecular Spectra' and afterwards follow the original papers of some leading worker in this field, tracing the development of his thought in recent years". With the publication of the eminently readable report by the Physical Society referred to below, this difficulty should be resolved for many years.

Dr. W. Jevons is to be congratulated on the completion of a valuable piece of work. $\mathrm{He}$ has produced a model report, subsuming on the part of the general reader no previous specialised know. ledge of the subject and yet providing for the expert a most useful compilation of data. Within the limits which Dr. Jevons has prescribed for himself, he has written a clear and well arranged account of the subject for which physicists and chemists alike will wish to thank him; research workers will do so more especially for the valuable appendices he has included. Through the tables and classified bibliography given here there is now provided a rapid and reliable approach to the literature of almost all known band spectra of diatomic molecules.

\section{R. C. JoHnson.}

\section{International Auxiliary Languages*}

$\mathrm{I}^{\mathrm{N}}$ the pamphlets referred to below, Dr. Ritter gives us an excellent survey, with statistics, of the language situation of the world, and of the cogent reasons in favour of the adoption, by formal agreement, of some easily learned international language.

The complacent assurance, once almost universal, that the 'blessings' of industrial civilisation, and of 'English, well and clearly spoken', would rapidly extend to the whole of humanity, has given place to a sceptical attitude towards our own culture. A powerful reverse current has set in; many of our own eminent thinkers set the highest value upon a multitude of cultures, upon competition rather than fusion between them, even upon war itself. Innumerable students all over the world are being educated in languages, sometimes limited to one or two millions of people, which give them no access to English, French, and German literature. Those who are very good natural linguists may acquire all three of these, but the majority will acquire only one, and many, ungifted as linguists, will never

" "Die Sprache im internationalen Verkehr" (2nd ed., pp. 48) and national Labour Burcau, Geneva. Printed as MS., 1932. read easily in any but their mother tongue. A point worthy of strong emphasis is the fact that inability to acquire foreign languages is in no way a sign of mental inferiority generally.

Dr. Ritter points out that the above facts limit the field of selection for persons who are to assist in international co-operation, as in the League of Nations. It is obvious that discussions carried on through interpreters are severely handicapped. The same argument applies, of course, over much wider fields. Dr. Ritter pleads for the formation by the League of a commission to study the problem, and hopes for the endowment out of private funds of an institute as well. He also puts forward his own suggestions for a world language; a phonetic, orthographic, and grammatical simplification of Mr. Ogden's "Basic English". It is much altered, but readily intelligible to an English reader, as soon as the rules of simplification are grasped. It is somehow less of a shock than the efforts of our own more advanced spelling reformers, or Prof. Zacharisson's "Anglic". Those whose hearts are set upon the retention of stylistic elegancies, even in the statement of pure matters of fact, will not like it any better than Mr. Ogden's "Basic". 
Dr. Ritter is concerned solely with the necessity for an officially recognised international language. $\mathrm{He}$ believes that no living language stands a chance of adoption owing to jealousy, and also to more realistic considerations of commercial competition. Further progress along the lines of Esperanto and Ido seems unlikely; all the signs point to Basic English competing with them successfully on account of its superior simplicity and practicality, wherever these qualities are called for. Also, the fact that to learn it is to make a good beginning with learning standard English tells enormously in its favour.
Dr. Ritter's language (he has not named it) is somewhat easier to learn, since it has only 17 letter's, three pronouns, and no irregularities; but learning it means learning English wrongly, and that will operate against it. But Dr. Ritter believes that only so, as an invented language, would it stand a chance of adoption by the League.

Edison's first invention was an electric vote recorder for Congress, designed to expedite business. It was gently but firmly explained to him that this is the last thing in the world that politicians desire.

H. S. H.

\section{New Applications of the Cathode Ray Oscillograph}

$\mathrm{T}$ HE modern cathode ray oscillograph has now become a well-established tool of the physicist and electrical engineer for the experimental study of electromotive forces varying with time. In addition to its normal cscillographic use for the investigation of the wave-form of periodic electromotive forces, it has been extensively used for the study of transient or non-recurring phenomena, such as, for example, the reception of an atmospheric in a wireless aerial.

The electromotive force which operates the oscillograph may not, however, be the ultimate object of study; this force may be produced or derived in some manner from the phenomena which it is desired to investigate. It is well-known that certain reactions taking place in the human body, such as the beating of the heart, may be made to produce changes in the flow of current in an electrical circuit connected to the body in a suitable manner. The vibrating string galvanometer has been used extensively for this purpose in the past. It is quite probable, however, that the cathode ray oscillograph will find a useful application in this field shortly, since it has an advantage over the galvanometer in that it provides a continuously visible image of the derived electrical variations and the results of various experimental operations may be seen immediately on the oscillograph screen.

One of the difficulties accompanying the use of the cathode ray oscillograph in the past has been that of providing a suitable time-base for the spot of light forming the image on the fluorescent screen. In the June 1932 issue of the Journal of the Institution of Electrical Engineers, a description is given of a method of producing a circular time base as developed by the staff of the Radio Research Station, Slough. In this method, sine wave alternating voltages are applied in phase quadrature to the deflect. ing plates of the cathode ray oscillograph, with the result that a circular motion is imparted to the spot on the screen, the time of rotation of the spot being equal to the period of the alternating current supply. The electromotive force to be studied is introduced at a suitable point in the circuit and produces radial deflections of the spot from its circular path, without affecting the intensity or definition of the image. The time of recurrence or duration of the phenomenon under observation can be estimated from angular measurements on the screen, while the amplitude of the electromotive force is measured radially.

The paper referred to above mentions the possible application of the method to the comparison of highorder harmonic frequencies, and to the checking of the period of a seconds pendulum against an altern. ating current supply of accurately controlled frequency. In the latter case a rate of the pendulum corresponding to about $2 \frac{1}{2}$ seconds a day can be determined in a few seconds, while any departure from uniformity of the pendulum swing may be quickly studied.

\section{Heavy Current Circuit-Breakers}

$\mathrm{I}^{\mathrm{N}}$ most countries of the world the supply of towns and industrial districts with electrical energy is a matter of the greatest importance. The increasing size of central power stations and the extensive interconnexion of high tension networks, which in some cases spreads over the political frontiers of individual countries, makes the proper choice of protective epparatus essential to the safety of the system. One of the most troublesome faults arises when there is a short circuit in some part of the network. To counteract its effects apparatus to break the circuit, 'circuit-breakers', must be used in connexion with suitable relays. These must be capable of opening and closing on a short circuit several times consecutively without danger.

In the Brown Boveri Review for June last, $H$. Thommen describes the plant used in the Brown Boveri works in Baden for testing circuit-breakers. Prior to 1897 , knife-switches with air-breaks were employed to break the circuit, but they were unsuitable as they produced very long ares even at low pressures. C. E. L. Brown was the first to break the arc under oil and it was thought that this would give the perfect solution. It was found when breaking high power that oil circuit-breakers occasionally gave rise to serious disturbances. Sometimes the mechanical strength proved insufficient, or the opening distance of the contacts was too small so that flash overs to the casing resulted. Unsuitable contacts on heavy currents sometimes welded together and ruined the apparatus.

The very thorough tests carried out by the Brown Boveri Co. have cleared up many of the difficulties which lay in the way of designers. By means of slow-motion cinematography, they demonstrated the oil piston theory. According to this theory, rapidly growing bubbles form around the arc at rupture in the oil. The oil above the break is projected like a 